\title{
Brief Consultation to Families of Treatment Refusers with Symptoms of Obsessive Compulsive Disorder: Does It Impact Family Accommodation and Quality of Life?
}

\author{
Melanie M. VanDyke1,2, C. Alec Pollard ${ }^{1,3}$, Jacob Harper2, Kyle E. Conlon",4 \\ ${ }^{1}$ Center for OCD and Anxiety-Related Disorders, Saint Louis Behavioral Medicine Institute, St. Louis, MO, USA \\ ${ }^{2}$ Department of Liberal Arts, St. Louis College of Pharmacy, St. Louis, MO, USA \\ ${ }^{3}$ Department of Family and Community Medicine, Saint Louis University, St. Louis, MO, USA \\ ${ }^{4}$ Currently at the Department of Psychology, Stephen F. Austin State University, Nacogdoches, TX, USA \\ Email: Melanie.VanDyke@stlcop.edu
}

Received 30 July 2015; accepted 20 September 2015; published 23 September 2015

Copyright (C) 2015 by authors and Scientific Research Publishing Inc.

This work is licensed under the Creative Commons Attribution International License (CC BY). http://creativecommons.org/licenses/by/4.0/

(c) (i) Open Access

\section{Abstract}

Family members are often directly and significantly impacted by the restrictive demands of OCD, a frequently disabling disorder. Family accommodation behaviors (i.e., doing things for or because of the OCD sufferer that a person would not normally do) are associated with dysfunction, including poorer treatment responses in OCD sufferers and greater distress in family members. Although evidence suggests family-based intervention can reduce symptoms in OCD sufferers who participate in treatment, there is a lack of research documenting the impact of interventions designed for the families of OCD treatment refusers (TR). Brief Family Consultation (BFC) was developed by our clinical team to help families refocus their efforts on the things that they can realistically control and change (e.g., participation in compulsions). In this crossover study, twenty families related to an individual who exhibited OCD symptoms but had refused treatment were assigned to five phone sessions of either BFC or a psychoeducation condition. Compared to this credible, attention-placebo control group (Brief Educational Support; BES), BFC (but not BES) resulted in reductions in family accommodation behavior, yet neither BFC nor BES resulted in improved quality of life for family members of treatment refusers. BFC is one of the first interventions to be evaluated for its ability to help families when their loved ones with obsessive compulsive symptoms refuse treatment. This pilot study provides new insights for clinicians and researchers to better address the needs of these neglected families. 


\section{Keywords}

\section{OCD, Treatment Refusal, Family Therapy, Family Accommodations, Quality of Life}

\section{Introduction}

Obsessive-Compulsive Disorder (OCD) is a debilitating psychiatric disorder characterized by recurrent obsessions or compulsions that cause marked distress and significantly interfere with a person's daily life (American Psychiatric Association, 2013). Common obsessions include repeated, intrusive thoughts about contamination, repeated thoughts about causing harm to others, and a preoccupation with arranging things in a specific order; Compulsions include excessive hand washing, organizing, and checking (for a review, see Franklin \& Foa, 2014). OCD is associated with impairments in occupational and interpersonal functioning (e.g., Markarian et al., 2010) and lower quality of life (Fontenelle et al., 2010; Kugler et al, 2013). Recent guidelines for treating OCD (Koran \& Simpson, 2013) reflect that numerous studies demonstrate that exposure and response prevention (ERP; a form of cognitive behavioral therapy) is effective for treating OCD (Rosa-Alcazar, Sanchez-Meca, Gomez-Conesa, \& Marin-Martinez, 2008), and improving the quality of life among outpatients treated for OCD (Diefenbach, Abramowitz, Norberg, \& Tolin, 2007; Weidle, Ivarsson, Thomsen, Lydersen, \& Jozefiak, 2014). However, the majority of individuals with OCD delay treatment, or worse, do not seek treatment (Garcia-Soriano, Rufer, Delsignore, \& Weidt, 2014).

While considerable research has focused on individuals with OCD, significantly less research has investigated the family members of OCD sufferers. Mounting empirical evidence suggests that OCD presents a tremendous burden for families, causing emotional strain and disrupting the daily routines of family members (Steketee, 1997). Other studies (e.g., Black, Gaffney, Schlosser, \& Gabel, 1998; Cooper, 1996) suggest that family members often become frustrated with their relative's OCD behavior, resulting in a significant amount of familial tension and conflict. Furthermore, many family members attempt to conceal their relative's OCD in order to avoid the social stigma of mental illness, which may hinder the family's ability to form satisfying and lasting social relationships (Stengler-Wenzke, Trosbach, Dietrich, \& Angermeyer, 2004). Not surprisingly, significant family burden can result in a reduced quality of life for families of people with OCD (Stengler-Wenzke, Kroll, Matschinger, \& Angermeyer, 2006). Overall, families of OCD sufferers experience a considerable amount of stress when attempting to manage the challenges associated with their relative's disorder.

One important aspect of the relationship between family members and OCD sufferers is how family members respond to the symptoms of OCD. Van Noppen and her colleagues (1997a) reported that family members often became involved in their relative's ritualistic behaviors, either in assisting to satisfy the OCD symptoms or in resisting and refusing to participate in the OCD symptoms. For instance, a family member may use disposable dishes, buy extra soap, or wash items repeatedly for an OCD sufferer with contamination fears. Research indicates that family members who modify their behavior to accommodate obsessive-compulsive symptoms experience greater family distress (Calvocoressi et al., 1999). In order to placate their relative's OCD demands and reduce possible familial conflict, families often modify their routines and provide reassurance to make the OCD sufferer comfortable. Although such accommodations are understandable and common, family accommodations are positively correlated with OCD symptom severity (Gomes et al., 2014) and negatively correlated with global functioning (Calvocoressi et al., 1999). Furthermore, OCD sufferers with accommodating families may experience increased OCD symptoms (Thompson-Hollands, Edson, Tompson, \& Comer, 2014) because OCD suffers feel that their beliefs and behaviors are legitimized by their families. Therefore, patients whose families accommodate their symptoms have lower response rates to behavioral therapy (Amir, Freshman, \& Foa, 2000; Thompson-Hollands, Edson, Tompson, \& Comer, 2014). Family treatments designed to decrease family accommodations have been shown to help patients with severe OCD symptoms (Thompson-Hollands, Abramovitch, Tompson, \& Barlow, 2015). Finally, greater family accommodation behaviors are related to increased family caregiver stress (Calvocoressi et al., 1999), as well as depression and anxiety in family members (Amir, Freshman, \& Foa, 2000).

Although inclusion of family members can reduce accommodation behavior in families of OCD sufferers participating in treatment, little is known about how to help the families of treatment refusers (TR). While some of 
the treatment barriers for sufferers have been identified (Gentle, Harris, \& Jones, 2014), there have been few studies, if any, that have systematically examined the families of TR with OCD. Families of TR have additional challenges. These families may feel their only option is either to wait helplessly until the OCD sufferer pursues help or to continue trying to coerce a noncompliant individual into therapy. Often neither of these options produces a satisfactory outcome and the burden on family members persists.

In response to frequent requests for help from families of TR, a multidisciplinary team of clinicians at the Center for OCD and Anxiety-Related Disorders (COARD) at Saint Louis Behavioral Medicine Institute developed an intervention called Brief Family Consultation (BFC) to address the needs of those impacted by a loved one with OCD who did not seek help. COARD specializes in treating patients with treatment resistant OCD (e.g., VanDyke \& Pollard, 2005), but families of treatment refusers require a unique approach. BFC is an intervention designed to assist family members in refocusing their cognitive, emotional, and physical resources on aspects of their life they can realistically control and change, as opposed to focusing and exhausting their resources on changing the OCD sufferer.

This study compares the effectiveness of BFC to a placebo control condition (Brief Educational Support; BES) in reducing family accommodation behavior and improving quality of life for families of TR. Due to the destructive impact of family accommodations, interventions are needed to reduce this behavior. When comparing pre-treatment and post-treatment scores, the predicted outcome is that BFC, but not BES, will result in significant reductions in family accommodation behavior. Although extant research suggests that OCD negatively affects the QOL of relatives (Stengler-Wenzke et al., 2006), further research is needed to identify effective interventions for improving QOL. Thus, it is predicted that BFC, but not BES, will result in significant improvements in the quality of life of family members.

\section{Methods}

\subsection{Participants}

Participating family members (PFM) included family members of individuals with obsessive compulsive symptoms who were refusing treatment. Twenty families joined the study, with a range of 1 - 3 people per family (13 men and 18 women), and these families had been impacted by the TR's OCD symptoms for an average of 15.89 years $(S D=9.94$; range 2 - 35 years). PFM were predominantly Caucasian $(93.5 \%)$ and lived in the United States: with 7 families in the Midwest, 5 in the East, 5 in the West, and 3 in the South, and they were related to the TR as the parents (15), spouses (9), siblings (4), or adult children (3). On average, PFM spent 18.41 hours/ week with the TR $(S D=30.31$; range 0 - 128 hours, with the majority of PFM living with the TR $(52.9 \%)$. On average, PFM's felt moderately angry or frustrated with the TR $(\mathrm{M}=2.16$ on a $0-4$ scale; $S D=1.12)$; however, some PFM reported no anger or frustration (15.8\%) and others reported severe levels of these negative emotions (52.6\%). The PFM described OCD symptoms in the TR that included washing (65\%), checking (50\%), hoarding (45\%), and other rituals (45\%) (Data were collected prior to hoarding being classified separately in DSM-5; American Psychiatric Association, 2013). Study consultants had at least 10 years of experience in treating OCD and families. They consulted with the PFM over the phone in five sessions over a seven week period and were assessed at several stages: 1) before the start of treatment, 2) one month after treatment, 3) one month after the second treatment, and 4) one year following the completion of their final treatment.

Inclusion criteria for PFM included being a family member of an individual who 1) exhibited symptoms consistent with an OCD diagnosis, 2) had not sought treatment in the last six months, and 3) had verbally refused to seek treatment or exhibited no treatment-seeking behaviors despite being informed about available treatment. Family member status was defined as people who were related by blood or marriage, including spouses and individuals who have a comparable relationship to an OCD sufferer (i.e., domestic partners). Exclusion criteria for PFM included a history of diagnosed psychotic symptoms, thought disorders, or significant cognitive impairments, as well as individuals with evidence of active alcohol or drug dependences. Children (under age 18) were excluded because the measures used were developed to measure adult functioning and the interventions were designed for adult family members.

\subsection{Procedure}

\subsubsection{Enrollment}

PFM were recruited by advertisements in the Obsessive-Compulsive Foundation's (currently the International 
OCD Foundation) and Anxiety Disorders Association of America's (currently the Anxiety and Depression Association of America) newsletters. PFM received consultation at no cost, and no additional incentives were offered for participation. The initial assessment for participation involved a brief telephone interview and written questionnaires about their quality of life and the level of participation in the OCD sufferer's symptoms. The study procedures were approved by the IRB at Saint Louis University.

\subsubsection{Treatment Conditions}

Participants scheduled five phone consultation sessions with a therapist experienced in the treatment of OCD. During these sessions, the therapist and PFM discussed ways to cope with the TR's behavior. One month after consultation sessions were completed, PFM were asked to complete the questionnaires about their quality of life, their participation in the TR's symptoms, and credibility and alliance treatment measures. PFM were debriefed by phone and were offered to participate in the alternative treatment condition (i.e., consultation or education) with their previous therapist. All PFM were asked to complete long-term follow-up, at least one year after their participation $(N=15)$.

The families were assigned using alternating, sequential assignment to either the Brief Family Consultation (BFC) or the Brief Educational Support (BES) condition. A coin toss (heads BFC, tails BES) decided whether the first family was assigned to BFC or BES. Subsequent families were then assigned to their initial treatment condition in an alternating manner to a group based on order of recruitment (defined by when the initial assessment packet was received). This method was chosen because the study had a small number of families, and sequential assignment ensures an equal number of families in each initial group. Ten families began in each group (with 1 - 3 PFM per family): 17 PFM were in the BFC group and 14 were in the BES group. Using a Crossover design, participating families were invited to complete the alternative condition following completion of the initial consultation condition. This methodology produced a combined total of 14 families completing BFC and 11 families completing the BES condition.

Brief Family Consultation (BFC). The goal of BFC is to normalize the family by focusing PFM on their own behavior, making behavioral changes that PFM can realistically control and that PFM are willing to implement. BFC draws upon cognitive behavioral and problem-solving models. PFM create and implement a Family Recovery Plan, designed to reduce PFM accommodation behaviors and address the TR's potential sabotage behaviors. PFM read "Someone I care about is not dealing with his OCD: What can I do about it?" from the OCD Newsletter article (Pollard \& Pollard, 2002). Each BFC session consisted of reviewing previous homework, discussing the next step of the recovery plan with the consultant, and planning for the next behavioral assignment. The emphasis was upon reducing the negative impact of OCD on the PFM and included the following components: 1) assessment of the ways in which each PFM copes with and is affected by the OCD sufferer's behavior; 2) cognitive restructuring to modify family beliefs that may interfere with their ability to develop realistic, attainable goals; 3) education about the nature of recovery avoidance and the counterproductive ways (e.g., accommodating, minimizing) in which families cope with OCD; and 4) coping, assertion, and contingency management skills training designed to reduce the negative impact of OCD on the family.

Brief Educational Support (BES). BES was developed as a credible placebo control condition for BFC using Van Noppen and colleagues' (1997b) “Learning to Live with OCD”, a pamphlet from the International OCD Foundation as a resource. The emphasis of BES was for the PFM to serve as an educational resource for the TR. BES focused on teaching the PFM about OCD, using psychoeducational material about the nature and treatment of OCD. This treatment condition included reading about OCD, discussing the information with the consultant, and leaving the information for the TR. PFM learned about key aspects of OCD, including four components: 1) the types of obsessions and compulsions associated with OCD; 2) suspected etiologies of OCD; 3) functional impairment in OCD (e.g., impairments in social, occupational/academic, and self-care activities); and 4) various empirically-supported treatment options for OCD. Each BES session consisted of a presentation by the therapist of an OCD topic, a discussion between the therapist and the PFM about this information, and finally, the family relaying information learned to the TR.

\subsection{Measures}

\subsubsection{Presence of OCD Symptoms and Treatment Refusal Status}

During the initial telephone interview, PFM described observable OCD symptoms of the TR using the OCD section of the Anxiety Symptom Inventory, a diagnostic screening instrument developed by our clinic based on 
DSM-IV criteria for OCD (American Psychiatric Association, 2000). The goal of this description is to establish OCD symptoms in the TR, not diagnose OCD. PFM were also asked about the TR's most recent contact with a mental health professional. PFM were asked about their willingness to participate and for their mailing address to send out the informed consent and other treatment measures.

\subsubsection{Family Accommodations Scale for OCD}

This study used a 12 item self-report version of the Family Accommodations Scale (FAS) for OCD, adapted from the original FAS Interviewer-Rated version. This clinician-administered interview (FAS-IR; Calvocoressi et al., 1999) demonstrated excellent inter-rater reliability (intraclass correlation coefficients range of 0.75 - 0.99 ) and good internal consistency (Cronbach's alpha coefficient of 0.82). The FAS used in this study is largely consistent with a recently developed self-rated version (FAS-SR; Pinto, Van Noppen, \& Calvocoressi, 2013), which demonstrated excellent internal consistency (Cronbach's alpha coefficient of 0.90) and strong agreement with the Interviewer-Rated version (intraclass correlation coefficient of 0.78 ).

\subsubsection{Quality Of Life Inventory}

The Quality of Life Inventory (QOLI; Frisch, Cornell, Villanueva, \& Retzlaff, 1992) measures an individual's satisfaction with 17 areas of their functioning. The QOLI has alpha coefficients ranging from 0.77 to 0.89 and test-retest correlations of 0.80 to 0.91 . The QOLI is correlated with measures of subjective well-being ( 0.41 to 0.55 ) and measures of general psychopathology ( -0.44 to -0.45 ; Frisch, Cornell, Villanueva, \& Retzlaff, 1992).

\subsubsection{Credibility Expectancy Questionnaire}

Treatment measures included the Credibility Expectancy Questionnaire (CES; Devilly \& Borkovec, 2000), a standard measure for examining the credibility and expected benefit of treatment. The internal reliability and test-retest reliability were good. This treatment measure was administered to ensure equivalence between the treatment conditions.

\subsubsection{Working Alliance Inventory}

The second treatment measure was the client version of the Working Alliance Inventory-Short Form (WAI-SF; Tracey \& Kokotovic, 1989), a 12-item measure of therapeutic relationship with good reliability and adequate reliability. This treatment measure was also administered to ensure equivalence between the treatment conditions.

\section{Results}

\subsection{Preliminary Analyses}

No pretreatment difference were noted between initial groups on the FAS $(t(27)=0.817 ; p=0.421)$ nor on the QOLI $(t(27)=0.067 ; p=0.947)$. No post-treatment differences were noted between the initial treatment conditions on the CES $(t(16)=0.904 ; p=0.379)$ nor on the WAI-SF $(t(18)=1.455 ; p=0.163)$. These preliminary analyses used independent samples t-tests within the Statistical Package for the Social Sciences (SPSS) software program.

\subsection{Family Accommodation of OCD}

The average initial score on the FAS was $21.51(S D=13.04)$, indicating the tendency for PFM to accommodate the treatment refusers' OCD symptoms. PFM who completed BES did not show reduced accommodations ( $t$ (12) $=0.000 ; p=1.000$ ) with an average pre-post reduction of 0.00. PFM assigned to BFC (as their first or second treatment condition) had significantly lower FAS scores $(t(22)=3.562$; $p=0.002)$ with an average pre-post reduction of 7.00. One year after the consultation, PFM assigned to BFC (attending 2 - 5 sessions) maintained these improvements in family accommodation behavior $(t(12)=2.725 ; p=0.018)$, compared to their initial FAS scores. Changes in FAS scores over the course of the intervention were computed using paired-samples ttests within SPSS' software program.

\subsection{Quality of Life}

The average initial score on the QOLI was $2.31(S D=1.64)$, indicating the quality of life of PFM. There were no post-treatment differences in QOLI scores. PFM assigned to BFC $(t(20)=0.103 ; p=0.919)$ and PFM as- 
signed to BES $(t(12)=-0.640 ; p=0.534)$ had similar pre- and post-treatment scores on the QOLI. One year after the consultation, PFM assigned to BFC (attending 2 - 5 sessions) failed to show improvement in their overall quality of life ( $t(13)=-0.525 ; p=0.608)$, compared to their initial FAS scores. Changes in QOLI scores over the course of the intervention were computed using paired-samples t-tests within SPSS' software program (Table 1).

\section{Discussion}

Results suggest BFC can reduce accommodation of OCD behavior in the families of treatment refusers. Compared to the BES condition, BFC resulted in greater reductions in scores on the FAS, and these improvements were sustained one year after consultation. The clinical significance of this result is reflected by the FAS scores: pre-BFC scores can be characterized by moderate accommodations/2-3 times per week, and post-BFC scores can be characterized as mild accommodations/once per week. The brief, telephone format of our consultation makes this result especially notable. Researchers have proposed that interventions to address accommodations are needed (Gomes et al., 2014). Patients completing ERP had better treatment responses when their families participated in one such targeted intervention (Thompson-Hollands, Abramovitch, Tompson, \& Barlow, 2015). Results of the current study provide preliminary evidence that reduction of maladaptive accommodation behavior can occur in families of individuals with untreated symptoms. Though not the primary focus of this investigation, reducing family accommodations has the potential to increase a TR's incentive to pursue treatment. However, only two of the 20 families reported that the TR received treatment during their participation in the present study.

Neither of the treatment conditions was associated with improvements in quality of life. Robust gains in quality of life are difficult to achieve, and the brief format of the study may not have been long enough to impact quality of life in a meaningful way. Therefore, there was no change in quality of life one month after completing either the BFC or BES group. On average, both groups reported that they were generally satisfied, with high levels of quality of life on the QOLI both at the beginning and the end of the consultation. The QOLI scores are comparable to scores of general undergraduates not seeking treatment $(\mathrm{M}=2.63, S D=1.11)$; Frisch, Cornell, Villanueva, \& Retzlaff, 1992). PFM's scores do not fit our previous clinical experience. This inconsistency could be due to PFM being different from families members who are patients in our clinic, and PFM may be less impacted by OCD than our typical patients. Alternatively, the PFMs may have minimized their own difficulties and emphasized the TR's OCD behaviors. This focus on the OCD sufferer as "the sick one who needs treatment" is common in families of TRs-a pattern so typical that it was the subject of the OCD Newsletter entitled, "Families of OCD sufferers seldom get the help they need: Why they don't and why they should" (Pollard \& Pollard, 2006). In fact, throughout the project, PFM needed to be directed back to their own experiences, as they would drift to describing the TR.

Table 1. Impact of consultation conditions on family accommodation and quality of life measures.

\begin{tabular}{cccccccc}
\hline \multirow{2}{*}{ Measure } & \multicolumn{5}{c}{ Time Period } \\
\cline { 2 - 3 } & Initial Assessment & Pre-BFC & Post-BFC & Pre-BES & Post-BES & Follow-Up \\
\hline FAS & & & & & & \\
\hline Mean & 21.51 & 21.18 & $14.17^{*}$ & 21.08 & 21.08 & $16.57^{*}$ \\
SD & 13.04 & 13.89 & 9.87 & 13.92 & 16.68 & 11.64 \\
Range & $1-45$ & $3-44$ & $3-35$ & $3-45$ & $1-44$ & $3-36$ \\
$N$ & 29 & 23 & 23 & 13 & 13 & 13 \\
\hline QOLI & & & & & 2.28 & 2.71 \\
\hline Mean & 2.34 & 2.53 & 2.50 & 1.77 & 1.87 & 1.57 & 1.11 \\
SD & 1.61 & 0.96 & $-3.38-4.44$ & $-3.00-4.38$ & $-2.25-3.69$ & $0.63-4.13$ \\
Range & $-3-5.75$ & $0.11-3.69$ & 21 & 13 & 13 & 14 \\
\hline
\end{tabular}

\footnotetext{
${ }^{*} \mathrm{p}<0.05$
} 


\section{Limitations and Future Directions}

This preliminary data provides perhaps the first examination of a treatment designed for families of OCD treatment refusers; however, the present study included a limited sample size. Funding for this pilot study was insufficient to include more than 20 families. Also, some families dropped out or elected not to continue in the second condition ( 5 of 19 families dropped out of BFC; 0 of 11 families dropped out of BES; 1 family elected not to start BFC, 9 families elected not to start BES). In a similar study about family-centered treatment of hoarding, the attrition rate was as high as $44 \%$ ( 4 of 9 families), with low-rates of commitment suspected as the main factor in the high dropout rate, perhaps due to the length of treatment (14 sessions; Chasson, Carpenter, Ewing, Gibby, \& Lee, 2014). Our enrollment in the second treatment condition was uneven: the majority of families who completed BES first elected to continue the study with BFC (9 of 10 families), whereas the majority of families who completed BFC first elected not to continue with BES (9 of 10 families). Multivariate approaches (possible only with the inclusion of additional families) would help to clarify these order effects, as well as other variables such as the amount of contact that PFM have with the TR. Future studies should include a larger sample size and implement strategies to enhance completion rates.

An additional limitation of the present study is the limited number of sessions in the two treatment conditions. The protocol developed for this investigation included fewer sessions than our clinical team typically provided when BFC was administered in the clinic. Our effort to maximize cost-effectiveness and work within the limitations of funding may have jeopardized efficacy. Future research of this family intervention approach should examine protocols that include more sessions; however, this increase in sessions will have to be balanced with the concern over having too many sessions for families to complete, highlighted by Chasson and colleagues (2014).

There is no reason to believe that the efficacy of BFC is limited to OCD. Additional research is needed to study the usefulness of these methods with other disorders. For instance, with hoarding disorder being a newly specified disorder (American Psychiatric Association, 2013), future research is planned to investigate if different methods are needed for families with a person who hoards items and refuses treatment. In our clinical experience, similar methods have been useful with families of TR with social anxiety, panic disorder, and posttraumatic stress disorder.

Although one strength of BFC is its ability to adapt to each family's situation, this flexibility also results in variations between families in specific interventions utilized. These variations could make it more difficult to determine the essential, active ingredients of BFC, as well as complicating dissemination. A treatment manual, to share with researchers and practitioners faced with the families of TR, is currently in development. This manual will provide descriptions of treatment modules to address a variety of issues families of TR face. It is hoped that this more extensive description of modifications for specific family situations may optimally help these often neglected family members.

\section{Conclusion}

This pilot study helps to shed light on the often ignored families of OCD treatment refusers. Due to the negative effect of understandable but unhelpful family reactions to OCD symptoms, it is promising that brief interventions can change accommodation behaviors. However, BFC does not influence the quality of life for PFM. Therefore, new treatments are necessary to meet the needs of family members who are impacted by a loved one's OCD symptoms. Likely, these methods will include extending the number of sessions, while aiming to address the specific needs of individual family situations. Future research to evaluate these treatment methods will inform therapists who are asked "What can I do when my loved one will not get help?".

\section{Acknowledgements}

The authors would like to thank our research assistants, Dan Ferland, PhD and Jennifer Summers, PsyD, and study therapists, Heidi Pollard, MSN and Gary Mitchell, LCSW, at the Center for OCD and Anxiety Related Disorders at Saint Louis Behavioral Medicine Institute. We also thank our statistical consultant, Claude Gaebelein, PhD, and writing consultant, Susan Mueller, at St. Louis College of Pharmacy. Finally, we thank the International OCD Foundation for providing funding for this pilot study. 


\section{References}

American Psychiatric Association (2000). Diagnostic and Statistical Manual of Mental Disorders (4th ed., Text Revision). Washington DC: American Psychiatric Association.

American Psychiatric Association (2013). Diagnostic and Statistical Manual of Mental Disorders (5th ed.). Washington DC: American Psychiatric Association.

Amir, N., Freshman, M., \& Foa, E. B. (2000). Family Distress and Involvement in Relatives of Obsessive-Compulsive Disorder Patients. Journal of Anxiety Disorders, 14, 209-217. http://dx.doi.org/10.1016/S0887-6185(99)00032-8

Black, D. W., Gaffney, G., Schlosser, S., \& Gabel, J. (1998). The Impact of Obsessive-Compulsive Disorder on the Family: Preliminary Findings. Journal of Nervous and Mental Disease, 186, 440-442. http://dx.doi.org/10.1097/00005053-199807000-00010

Calvocoressi, L., Mazure, C. M., Kasl, S. V., Skolnick, J., Fisk, D., Vegso, S. J., Price, L. H. et al. (1999). Family Accommodation of Obsessive-Compulsive Symptoms: Instrument Development and Assessment of Family Behavior. Journal of Nervous and Mental Disease, 187, 636-642. http://dx.doi.org/10.1097/00005053-199910000-00008

Chasson, G. S., Carpenter, A., Ewing, J., Gibby, B., \& Lee, N. (2014). Empowering Families to Help a Loved One with Hoarding Disorder: Pilot Study of Family-as-Motivators Training. Behaviour Research and Therapy, 63, 9-16. http://dx.doi.org/10.1016/j.brat.2014.08.016

Cooper, M. (1996). Obsessive-Compulsive Disorder: Effects on Family Members. American Journal of Orthopsychiatry, 66, 296-304. http://dx.doi.org/10.1037/h0080180

Devilly, G. J., \& Borkovec, T. D. (2000). Psychometric Properties of the Credibility/Expectancy Questionnaire. Journal of Behavior Therapy and Experimental Psychiatry, 31, 73-86. http://dx.doi.org/10.1016/S0005-7916(00)00012-4

Diefenbach, G. J., Abramowitz, J. S., Norberg, M. M., \& Tolin, D. F. (2007). Changes in Quality of Life Following Cognitive-Behavioral Therapy for Obsessive-Compulsive Disorder. Behaviour Research and Therapy, 45, 3060-3068. http://dx.doi.org/10.1016/j.brat.2007.04.014

Fontenelle, I. S., Fontenelle, L. F., Borges, M. C., Prazeres, A. M., Rangé, B. P., Mendlowicz, M. V., \& Versiani, M. (2010). Quality of Life and Symptom Dimensions of Patients with Obsessive-Compulsive Disorder. Psychiatry Research, 179, 198-203. http://dx.doi.org/10.1016/j.psychres.2009.04.005

Franklin, M. E., \& Foa, E. B. (2014). Obsessive-Compulsive Disorder. In D. H. Barlow (Ed.), Clinical Handbook of Psychological Disorders: A Step-by-Step Treatment Manual (5th ed., pp. 155-205). New York: Guilford Press.

Frisch, M. B., Cornell, J., Villanueva, M., \& Retzlaff, P. J. (1992). Clinical Validation of the Quality of Life Inventory: A Measure of Life Satisfaction for Use in Treatment Planning and Outcome Assessment. Psychological Assessment, 4, 92101. http://dx.doi.org/10.1037/1040-3590.4.1.92

Garcia-Soriano, G., Rufer, M., Delsignore, A., \& Weidt, S. (2014). Factors Associated with Non-Treatment or Delayed Treatment Seeking in OCD Sufferers: A Review of the Literature. Psychiatry Research, 220, 1-10. http://dx.doi.org/10.1016/j.psychres.2014.07.009

Gentle, M., Harris, L. M., \& Jones, M. K. (2014). The Barriers to Seeking Treatment for Obsessive-Compulsive Disorder in an Australian Population. Behaviour Change, 31, 258-278. http://dx.doi.org/10.1017/bec.2014.20

Gomes, J. B., Van Noppen, B., Pato, M., Braga, D. T., Meyer, E., Bortoncello, C. F., \& Cordioli, A. V. (2014). Patient and Family Factors Associated with Family Accommodation in Obsessive-Compulsive Disorder. Psychiatry and Clinical Neurosciences, 68, 621-630. http://dx.doi.org/10.1111/pcn.12172

Koran, L. M., \& Simpson, H. B. (2013). Guideline Watch (March 2013): Practice Guideline for the Treatment of Patients with Obsessive-Compulsive Disorder. Arlington, VA: American Psychiatric Association.

Kugler, B. B., Lewin, A. B., Phares, V., Geffken, G. R., Murphy, T. K., \& Storch, E. A. (2013). Quality of Life in ObsessiveCompulsive Disorder: The Role of Mediating Variables. Psychiatry Research, 206, 43-49. http://dx.doi.org/10.1016/j.psychres.2012.10.006

Markarian, Y., Larson, M. J., Aldea, M. A., Baldwin, S. A., Good, D., Berkeljon, A. et al. (2010). Multiple Pathways to Functional Impairment in Obsessive-Compulsive Disorder. Clinical Psychology Review, 30, 78-88. http://dx.doi.org/10.1016/j.cpr.2009.09.005

Pinto, A., Van Noppen, B., \& Calvocoressi, L. (2013). Development and Preliminary Psychometric Evaluation of a SelfRated Version of the Family Accommodation Scale for Obsessive-Compulsive Disorder. Journal of Obsessive-Compulsive and Related Disorders, 2, 457-465. http://dx.doi.org/10.1016/j.jocrd.2012.06.001

Pollard, H. J., \& Pollard, C. A. (2002). Someone I Care About Is Not Dealing with His OCD, What Can I Do about It. OCD Newsletter, Summer, 1-5.

Pollard, H. J., \& Pollard, C. A. (2006). Families of OCD Sufferers Seldom Get the Help They Need: Why They Don't and Why They Should. OCD Newsletter, Spring, 14-15. 
Rosa-Alcazar, A. I., Sanchez-Meca, J., Gomez-Conesa, A., \& Marin-Martinez, F. (2008). Psychological Treatment of Obsessive-Compulsive Disorder: A Meta-Analysis. Clinical Psychology Review, 28, 1310-1325. http://dx.doi.org/10.1016/j.cpr.2008.07.001

Steketee, G. (1997). Disability and Family Burden in Obsessive-Compulsive Disorder. Canadian Journal of Psychiatry, 42, 919-928.

Stengler-Wenzke, K., Kroll, M., Matschinger, H., \& Angermeyer, M. C. (2006). Quality of Life of Relatives of Patients with Obsessive-Compulsive Disorder. Comprehensive Psychiatry, 47, 523-527. http://dx.doi.org/10.1016/j.comppsych.2006.02.002

Stengler-Wenzke, K., Trosbach, J., Dietrich, S., \& Angermeyer, M. C. (2004). Experience of Stigmatization by Relatives of Patients with Obsessive Compulsive Disorder. Archives of Psychiatric Nursing, 18, 88-96. http://dx.doi.org/10.1016/j.apnu.2004.03.004

Thompson-Hollands, J., Abramovitch, A., Tompson, M., \& Barlow, D. (2015). A Randomized Clinical Trial of a Brief Family Intervention to Reduce Accommodation in Obsessive-Compulsive Disorder: A Preliminary Study. Behavior Therapy, 46, 218-229. http://dx.doi.org/10.1016/j.beth.2014.11.001

Thompson-Hollands, J., Edson, A., Tompson, M., \& Comer, J. (2014). Family Involvement in the Psychological Treatment of Obsessive-Compulsive Disorder: A Meta-Analysis. Journal of Family Psychology, 28, 287-298. http://dx.doi.org/10.1037/a0036709

Tracey, T. J., \& Kokotovic, A. M. (1989). Factor Structure of the Working Alliance Inventory. Psychological Assessment: A Journal of Consulting and Clinical Psychology, 1, 207-210. http://dx.doi.org/10.1037/1040-3590.1.3.207

Van Noppen, B. L., Pato, M. T., \& Rasmussen, S. A. (1997b). Learning to Live with OCD: Obsessive Compulsive Disorder (4th ed.). Milford, CT: Obsessive Compulsive Foundation.

Van Noppen, B., Steketee, G., McCorkle, B. H., \& Pato, M. (1997a). Group and Multifamily Behavioral Treatment for Obsessive-Compulsive Disorder. Journal of Anxiety Disorders, 11, 431-436. http://dx.doi.org/10.1016/S0887-6185(97)00021-2

VanDyke, M. M., \& Pollard, C. A. (2005). Treatment of Refractory Obsessive-Compulsive Disorder: The St. Louis Model. Cognitive and Behavioral Practice, 12, 30-39. http://dx.doi.org/10.1016/S1077-7229(05)80037-9

Weidle, B., Ivarsson, T., Thomsen, P. H., Lydersen, S., \& Jozefiak, T. (2015). Quality of Life in Children with OCD before and after Treatment. European Child \& Adolescent Psychiatry, 24, 1061-1074. http://dx.doi.org/10.1007/s00787-014-0659-z 